\title{
Factores de incidencia de conductas ambientales negativas hacia las ciénagas de Bañó y Los Negros
}

\author{
Incidence Factors of Negative Environmental Behavior towards the Swamps of \\ Bañó and Los Negros
}

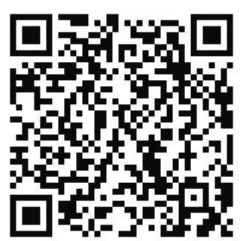

\author{
Jorge Villadiego-Lorduy \\ Fundación Saludmental para Todos \\ Córdoba, Montería, Colombia \\ villalord15@yahoo.es \\ Dennis Paul Huffman-Schwocho ${ }^{2}$ \\ Universidad de Chapingo \\ Texcoco, México \\ d_huffman@yahoo.com \\ Stalyn Yasid Guerrero-Gómez ${ }^{3}$ \\ Universidad Santo Tomas \\ Cundinamarca, Bogotá, Colombia \\ guerrerostalyn@hotmail.com \\ Adolfo Cortecero-Bossio ${ }^{4}$ \\ Kontiqui \\ Bolívar, Cartagena de Indias, Colombia \\ acobo71@yahoo.com \\ Sandra Rivero-Espitia \\ Corpoica \\ Cundinamarca, Bogotá, Colombia \\ Tatiana.rivero@gmail.com
}

Recibido 22 de julio de 2014 • Corregido 21 de mayo de 2015 • Aceptado 31 de julio de 2015

\footnotetext{
${ }^{1}$ Geógrafo. Estudiante del programa de Doctorado en Ciencias Naturales para el Desarrollo, Instituto Tecnológico de Costa Rica. Organización Social de Comunidades Negras "Nelson Mandela". Departamento de Córdoba, Montería, Colombia.

2 Doctor en Pedagogía. Profesor titular del programa de Doctorado en Ciencias Naturales para el Desarrollo.

${ }^{3}$ Estadístico. Profesor de la Universidad Santo Tomas. Departamento de Cundinamarca, Bogotá, Colombia.

${ }^{4}$ Ing. Químico. Msc. en Gestión Ambiental. Fundación Kontiqui. Departamento de Bolívar, Cartagena de Indias, Colombia.

${ }^{5}$ Zootecnista. Msc en Desarrollo Rural. Corpoica. Departamento de Cundinamarca, Bogotá, Colombia.
} 
doi: http://dx.doi.org/10.15359/ree.19-3.6

URL: http://www.una.ac.cr/educare

CORREO: educare@una.cr

Resumen. El propósito de este artículo es establecer los factores que están influyendo en conductas ambientalmente negativas por parte de los habitantes de los humedales de Bañó y Los Negros hacia estos. Para ello, se diseñó, validó mediante panel de expertos y aplicó un instrumento de investigación tipo cuestionario. Se establecieron, con base en el universo poblacional de 645 viviendas, dos tipos de diseño muestral para la aplicación del cuestionario -sistemático y aleatorio-, además de desarrollar su respectivo análisis estadístico, sin omitir el análisis de contenido de la información secundaria recolectada mediante fichas. Factores como el bajo nivel educativo, las escasas oportunidades de empleo y los pocos ingresos por núcleo familiar inciden de forma directa en el deterioro de los humedales. Casi un $50 \%$ de la población residente de los humedales no ha finalizado sus estudios básicos, por lo tanto, la capacidad de reflexión sobre las acciones que realizan los pobladores en los espejos de agua es poca, sobre todo cuando los ingresos económicos familiares son muy bajos (un 53\% de las familiar reciben ingresos menores a COL $\$ 10.000$ diarios). La escasa organización que presentan las comunidades ha contribuido a que no se desarrollen trabajos comunitarios articulados entre las poblaciones vecinas de los humedales y mucho menos alcanzar metas comunes. Por otra parte, la inoperancia de las autoridades ambientales tanto locales como nacionales ha favorecido la degradación de los ecosistemas, debido a la ineficiente gestión ambiental que han ejercido con el transcurrir del tiempo. Los humedales son la principal fuente de recursos naturales para estas comunidades y la permanencia de tales comunidades en el territorio está directamente relacionada con su conservación. La sinergia de los factores arriba mencionados ha desatado una problemática ambiental que se ha concretado en la disminución de la capacidad ecológica del humedal y de las condiciones ambientales del área de estudio.

Palabras claves. Deterioro ambiental, sinergia, pobreza, educación, gestión ambiental.

Abstract. The purpose of this article is to determine the factors that are influencing the environmentally negative behavior of the inhabitants of Bañó and Los Negros towards these wetlands. For this, a questionnaire was designed, validated by a panel of experts, and implemented. Based on a population of 645 households, we used two types of sample designs -systematic and random- to implement the research instrument. In addition to their respective statistical analysis, a content analysis was carried out with the secondary information collected using index cards. Factors such as low education, scarce employment opportunities, and low income per household have a direct impact on the deterioration of these wetlands. Almost $50 \%$ of the population living in the wetlands has not completed basic education. Thus, the possibilities to reflect on the actions done by people against the water mirrors are poor, especially when family incomes are very low ( $53 \%$ of the families receive incomes of less than COL $\$ 10,000$ a day). The scarce organization of the communities has prejudiced the development of joint activities between the neighboring communities of the wetlands, as well as the achievement of common goals. Furthermore, the ineffectiveness of local and national environmental authorities has contributed to the degradation of ecosystems due to an inefficient environmental management over the years. Wetlands are the main source of natural resources for these people and the permanence of such communities in the territory is directly related to the wetlands' conservation. The synergy of the above factors has unleashed environmental problems that have resulted in the reduction of the ecological capacity of these wetlands and the deterioration of environmental conditions in the area of study.

Keyword. Environmental deterioration, synergy, poverty, education, environmental management. 
El ser humano hoy día está obligado a ser ciudadano del mundo y habitante del Universo, hay que recordar que los ecosistemas no tienen límites político-administrativos.

Jorge Villadiego Lorduy

Los problemas ambientales de origen antrópico son el resultado de la mala interacción del ser humano con el medio, el planeta tiene límites ecológicos y el actual modelo económico capitalista ha contribuido a generar una problemática ambiental en las diferentes escalas territoriales, debido a la demanda constante por recursos naturales para generar procesos productivos; como resultado de tales procesos las sociedades obtienen bienes y servicios y se generan desechos. Desde los años 70, con el club de Roma y el estudio desarrollado y titulado los límites de crecimiento (Meadows, Randers, Meadows y Behrens (1972) se viene advirtiendo a la humanidad que, de seguir con el modo de vida actual, se estará yendo hacia un abismo sin retorno.

La crisis del medio ambiente se ha ido acelerando durante la segunda mitad de este siglo junto con la expansión capitalista. En última instancia, los procesos socioeconómicos y tecnológicos desencadenantes de la crisis ambiental, se unen a la incapacidad de comprensión humana del ambiente, del mundo y de la vida en su compleja totalidad, para admitir la verdadera dimensión del hombre en la naturaleza. (Colín, 2003, p. 104)

Una medida o mecanismo que desde la gestión ambiental se puede utilizar para disminuir el impacto de tipo negativo que realiza el ser humano al ambiente es la educación ambiental. ÁlvarezyVega (2009) indican quela ciudadanía necesita"adquirir urgentemente un conocimiento (alfabetización científico-ambiental) y un comportamiento'ecológico' que permita desarrollarse sin crecer más allá de... los límites y desarrollar una nueva cultura intelectual, de consumo y tecnológica. En ello la educación, entendida como una 'educación transformadora orientada hacia la sostenibilidad', tiene un papel fundamental" (p. 247).

Para poder generar un modelo educativo, en especial del tipo no formal y que contribuya a mejorar las condiciones ambientales de ecosistemas como lo son las ciénagas de Bañó y Los Negros, es vital identificar los factores que están incidiendo en los comportamientos negativos de los individuos hacia determinado entorno y, a la vez, a partir de un análisis de esos factores, plantear acciones de tipo educativo que coadyuven a mitigar esos comportamientos, y que propendan a fomentar actitudes ambientales positivas. Holahan (1991, p. 15) define las actitudes ambientales como "los sentimientos favorables o desfavorables que se tienen hacia alguna característica del medio o hacia un problema relacionado con él".

Desde la psicología ambiental se han desarrollado una serie de categorías que agrupan los factores que podrían influir en conductas ambientalmente negativas hacia el medio. Álvarez y Vega (2009) señalan los siguientes: 
doi: http://dx.doi.org/10.15359/ree.19-3.6

URL: http://www.una.ac.cr/educare

CORREO: educare@una.cr

1. Factores metodológicos: Relacionados con la medición de actitudes y conductas con un grado de especificidad similar. No se puede medir actitudes y conductas muy generales hacia el medio con medidas muy específicas de conducta.

2. Factores contextuales: Relacionados con la relevancia, la relación coste-beneficio de la acción, la influencia de la publicidad, el tiempo transcurrido entre la evaluación de la actitud y la conducta, entre otros.

3. Factores psicosociales: Que se refieren a variables y representacionales, tales como características disposicionales, autoritarismo, locus de control y grado de responsabilidad personal, considerados fuertes predictores de la actitud ambiental y, por consiguiente, de los comportamientos ambientalmente responsables.

4. Factores sociodemográficos: Como género, nivel de estudios, religión, ideología política, status socioeconómico, lugar de residencia cuya influencia sobre la realización de conductas proambientales no es concluyente, y que ofrecen, incluso, resultados contradictorios.

5. Factores cognitivos: En referencia a los conocimientos sobre el medio ambiente; sin embargo, Hwang, Kim y Jeng (2000) indican que el conocimiento sobre el medio ambiente, así como el grado de adscripción de responsabilidad de la persona ante la conducta, no son causas suficientes para la realización de conductas proambientales.

Álvarez y Vega (2009) enfatizan en que los individuos solo realizan conductas ambientalmente responsables cuando están suficientemente informados sobre la problemática ambiental, se encuentran motivados hacia ella y, además, se ven capaces de generar cambios cualitativos, están convencidos de la efectividad de su acción y de que esta no les generará dificultades importantes. Por lo tanto, la educación es el mejor camino para cambiar las actitudes y comportamientos de los individuos hacia el medio, solo así se formarán ciudadanas y ciudadanos preocupados por tener una relación armónica con su entorno.

La educación es necesaria para todo ser humano, pues bien orientada e integral puede servirle para interpretar su realidad, ya que relaciona sus distintos componentes y conforma un universo de posibilidades, aprende y sustenta su ubicación en la sociedad, en general, y de la vida, en particular. (Martínez, 2010, p. 100)

En el caso de las poblaciones vecinas a los humedales de Bañó y Los Negros, como son Cotocá Arriba, Palo de Agua y Castilleral, están ejerciendo fuertes presiones sobre estos ecosistemas, producto de una sumatoria de factores de orden económico, social, educativo, entre otros tantos, que han llevado al deterioro de las condiciones ambientales en el territorio 
y a la disminución de la calidad de vida de sus pobladores. Por eso, se hace necesario generar procesos educativos que contribuyan a mitigar tales problemas $y$, de paso, a concretar las potencialidades del territorio.

\section{Materiales y métodos}

El método que se utilizó para determinar los factores que están influyendo de forma negativa en los habitantes de las Cienagas de Bañó y Los Negros se soportó en el análisis de información primariay secundaria. En términos de información secundaria se recopiló información que permitiera establecer aportaciones teóricas de aspectos conductuales de individuos y comunidades hacia el medio. Para el caso del estudio de la información primaria se diseñó y aplicó un instrumento de investigación tipo cuestionario que buscó recopilar información de variables socioeconómicas, de problemas y conflictos ambientales y de percepción y cultura ambiental de habitantes del área de interés. Lo anterior, con el fin de contrastar la información secundaria analizada con las visitas a campo realizadas y las respuestas obtenidas con la aplicación del instrumento. La población universo de la investigación estuvo determinada por 645 viviendas habitadas pertenecientes a los corregimientos de Cotocá Arriba (110), Castilleral (146) y Palo de Agua (389). Se determinó un diseño de muestreo por estrato, donde los estratos corresponden a los diferentes pueblos, para Cotocá Arriba y Castilleral se les aplicó un muestreo sistemático y en el caso de Palo de Agua se implementó un muestreo aleatorio simple. Se aplicó un total de 72 encuestas de 40 ítems por cuestionario, distribuidos de la siguiente forma: Cotocá Arriba y Castilleral 12 cada una, Palo de Agua 48. Los diseños implementados fueron utilizados debido a que eran los que mejor se ajustaban a la organización espacial del pueblo y a la limitación de recursos disponibles. Igualmente, para el procesamiento y análisis de la información recolectada de la aplicación del cuestionario se emplearon métodos de estadística inferencial basada en el cálculo de estimaciones porcentuales y valores absolutos. La información del total de viviendas existentes a la fecha y sobre la cual se trabajó el diseño muestral se obtuvo de los líderes comunitarios de cada uno de los pueblos y de la verificación en campo de las viviendas habitadas. El software con el que se trabajó el análisis estadístico fue R versión 3.0.2.

\section{Resultados y discusión}

\section{Factores que inciden en conductas ambientalmente negativas hacia los humedales}

Los humedales de Bañó y Los Negros se encuentran ubicados en el bajo Sinú, exactamente entre los corregimientos de Cotocá Arriba, Castilleral y Palo de Agua, todos pertenecientes al área rural del municipio de Lorica, departamento de Córdoba, Colombia. La primera está localizada a $09^{\circ} 08^{\prime} 09^{\prime \prime} \mathrm{N}$ y $075^{\circ} 50^{\prime} 12^{\prime \prime} \mathrm{W}$, la segunda a $09^{\circ} 07^{\prime} 59^{\prime \prime} \mathrm{N}$ y $075^{\circ} 50^{\prime} 49^{\prime \prime} \mathrm{W}$ (Ver figura 1 ). 
doi: http://dx.doi.org/10.15359/ree.19-3.6

URL: http://www.una.ac.cr/educare

CORREO: educare@una.cr

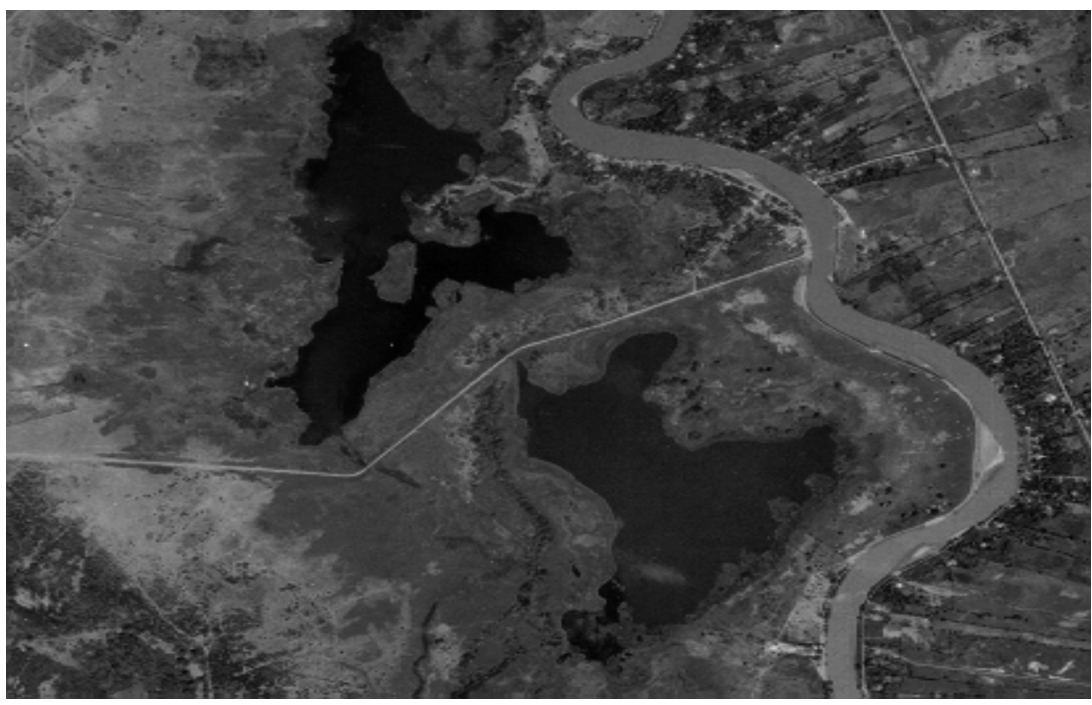

Figura 1. Fotografía aérea de las ciénagas de Bañó y Los Negros, margen izquierdo del río Sinú, en el corregimiento de Cotocá, municipio de Lorica. Ciénaga de Bañó (parte inferior en la foto) y ciénaga Los Negros (parte superior en la foto). Instituto Geográfico Agustín Codazzi (1994)6.

Las ciénagas de Bañó y Los Negros tienen como primeros vecinos a las poblaciones de Cotocá Arriba, Palo de Agua y Castilleral. Estas tres poblaciones son las que tienen mayor influencia en los territorios de la ciénagas; sin embargo, se puede mencionar las comunidades de Garavito, Guamal, Mompox y Sarandelo, entre otras, las cuales ejercen presión sobre los humedales de manera esporádica. La población de los tres corregimientos se estima a 2014 en 3182 habitantes de los cuales 2114 son personas adultas (66,4\%), 557 niños y niñas (17,5\%) y 489 adolescentes (15,4\%).

Hoy, la problemática ambiental de Bañó y Los Negros se concreta en 5 problemas: A) Desecación de los humedales. B) Pérdida de la biodiversidad. C) Manejo irracional de residuos. D) Contaminación y degradación del recurso suelo y agua. E) Deficientes condiciones sanitarias. Lo anterior se originado por la aparición de una serie de factores que han influenciado de forma negativa en el accionar de las poblaciones que habitan el humedal. Dentro de los factores que se identificaron con la aplicación del instrumento, las entrevistas realizadas a los líderes comunitarios, la revisión de información secundaria y del análisis mismo de la problemática el territorio se encuentran:

${ }^{6}$ Nota: El Instituto Geográfico Agustín Codazzi es el autor de la fotografía aérea de las ciénagas de Bañó y Los Negros, se señala que dentro de sus funciones se encuentra la de elaborar y vender a quien lo solicite la cartografía base de Colombia (Mapas, fotografías aéreas, etc.). 


\section{Bajos ingresos económicos y pocas oportunidades de empleo}

Según los valores proporcionados por el Departamento Nacional de Estadística (DANE, 2013) en su boletín Pobreza monetaria y multidimensional en Colombia 2012, se clasifica, de acuerdo con valores de ingreso por familia, que un hogar compuesto por 4 personas es pobre, si el ingreso total del hogar está por debajo de $\$ 808.332$ (USD 429.96). Si la familia vive en las cabeceras, este valor cambia a $\$ 892.604$; si vive en el resto (área rural) a $\$ 534.088$. En términos de la pobreza extrema si un hogar está compuesto en promedio por 4 personas; se clasificará como pobres extremos los hogares en donde su ingreso total esté por debajo de \$364.828. A nivel de las cabeceras, este valor cambia a $\$ 381.404$, en el resto (rural) $\$ 310.880$. Para el caso de las poblaciones aledañas a los humedales Bañó y Los Negros un $85 \%$ de sus habitantes se encuentra categorizados en pobreza y pobreza extrema. Se calculó que de las 645 viviendas habitadas, un 53\% (342) de las familias en el territorio reciben ingresos diarios menores a $\$ 10000$ (un poco mas de US\$ 5 en promedio reciben entre $\$ 5000$ a $\$ 7000$ por familia), 204 (32\%) familias tiene ingresos en un rango de $\$ 10.000$ a menos de $\$ 20550$ (un poco mas de US\$ 5 y menos de US\$11, se estaría hablando de un promedio $\$ 15000$ diarios). De lo anterior, se tiene que, debido a los bajos ingresos y pocas oportunidades de empleo en la zona, como alternativa económica le queda a las poblaciones vecinas desarrollar actividades productivas, las cuales, por su mal manejo, terminan afectando los humedales: la caza y tráfico de especies, tala de aboles con fines de uso energético, pesca intensiva y por fuera de tallas permitidas, pastoreo en los terrenos aledaños a las ciénagas, entre otras más.

Jiménez (1996, citado por Colín, 2003, p. 104) afirma que la causa mayor del deterioro continuo del medio ambiente global es el insostenible modelo de producción y consumo, particularmente en los países industrializados. En tanto que en los países en desarrollo la pobreza y la degradación ambiental están estrechamente interrelacionadas.

En los países en desarrollo, uno de cada cuatro hogares se encuentra en situación de pobreza. "El $40 \%$ de los hogares urbanos de África y el $25 \%$ de los hogares urbanos de América Latina vive por debajo de la línea de pobreza definida en cada país" (Galarza y Gómez, s. f., p. 4), (tabla 1).

Tabla 1

Ingresos por vivienda

\begin{tabular}{lccccc}
\hline \multicolumn{7}{c}{ Ingresos vivienda } \\
\hline Periodicidad & $<\$ 10000$ & $\$ 10000, \$<20550$ & $\$ 20550, \$ 41100$ & $>\$ 40100,<\$ 61650$ & $>\$ 61650$ \\
\hline Diariamente & 163 & 111 & 0 & 0 & 8 \\
Semanalmente & 106 & 50 & 47 & 12 & 0 \\
Quincenalmente & 28 & 20 & 0 & 0 & 0 \\
Mensualmente & 45 & 24 & 0 & 33 & 0 \\
\hline Total & 343 & 205 & 47 & 45 & 8 \\
\hline
\end{tabular}

Nota: Elaboración propia. 
doi: http://dx.doi.org/10.15359/ree.19-3.6

URL: http://www.una.ac.cr/educare

CORREO: educare@una.cr

\section{Bajo nivel de educación}

Para Álvarez (2003, citado por Martínez, 2010, p. 99), la educación se entiende como:

Un proceso de desarrollo socio-cultural continuo de las capacidades que las personas en sociedad deben generar y que se realiza tanto dentro como fuera de su entorno, a lo largo de toda la vida. La educación implica impulsar las destrezas y las estructuras cognitivas, que permiten que los estímulos sensoriales y la percepción del mundo-realidad se conviertan de información significativa, en conocimientos de su construcción y reconstrucción, así como en valores, costumbres, que determinan los comportamientos o formas de actuar.

Referente a las poblaciones aledañas a los humedales, se tiene que de las 645 viviendas habitadas, 295 (45,7\%) cuentan con algún individuo que tiene un nivel de estudios básicos incompletos, sea primaria o secundaria. Si se suma el $45,7 \%$ de estudios básicos incompletos con el $2,6 \%$ de personas iletradas se hablaría de un $48,3 \%$ de viviendas encuestadas, donde por lo menos un individuo no ha desarrollado de forma adecuada sus estudios básicos. El hecho de que existan tan bajos niveles educativos en el área de estudio puede afectar en el grado de entendimiento o conciencia que tengan habitantes vecinos de Bañó y Los Negros acerca de los impactos de tipo negativo que generan con sus acciones al entorno, aún teniendo estos el conocimiento empírico acerca de dicho tipo de ecosistemas, obtenido por largos periodos de habitar en el territorio; ejemplo de ello puede ser la caza y tráfico de especies que realizan en todo el año. Su nivel de conciencia no es tan alto para comprender que si siguen cazando y traficando con especies terminarán por extinguirlas.

Casi un $46 \%$ de los pobladores de los humedales no han desarrollado procesos de estudios básicos de forma adecuada, debido a diferentes factores como son los bajos recursos económicos, falta de material educativo, los mismos padres por el bajo nivel académico que poseen, la distancia hacia los centros educativos, entre otras causas, lo que ha ido contribuyendo al deterioro de la relación ser humano-medio. El hecho de que no se hayan desarrollado procesos eficientes de educación en casi el $50 \%$ de la población quiere decir que no ha habido un proceso de construcción, reflexión y adquisición de conocimientos, valores y desarrollo de competencias tanto individuales y colectivas (figura 2). Al respecto, Freire (1995, citado por García, Figueroa y Gómez, 2013, p. 202) señala que la educación, y sobre todo la ambiental, puede contribuir:

A una conciencia crítica e integral de nuestra situación en el planeta, ... es un agente importante en la transición a una nueva fase ecológica de la humanidad [y busca] comprender su relación en la biosfera humanizada, al formar personas capaces de interpretar y transformar el mundo, y de dar importancia a los derechos de todos los seres vivos (incluyendo humanos) y la naturaleza, para contribuir a plantear políticas y culturas basadas en necesidades a corto plazo. 


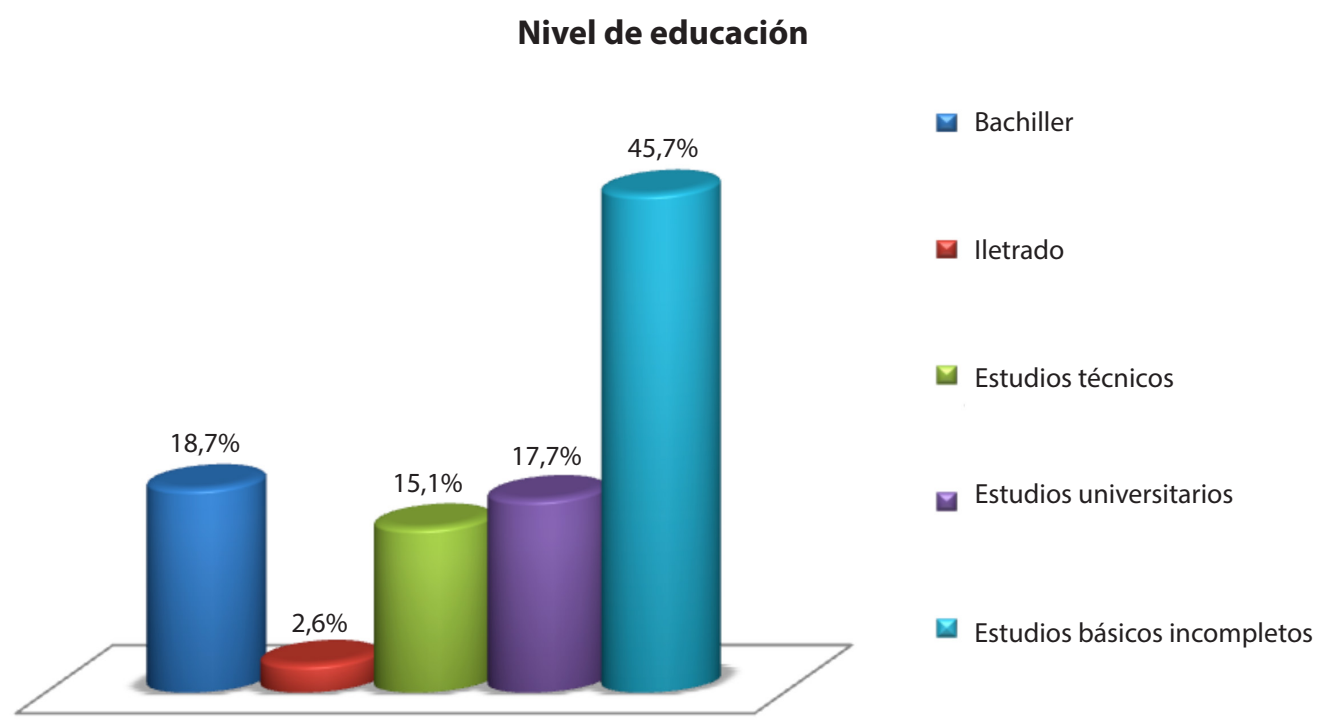

Figura 2. Nivel de educación de la población aledaña a los humedales. Elaboración propia.

\section{Estructura y organización comunitaria ineficiente}

A nivel de organización comunitaria, existen varias organizaciones no gubernamentales, dentro de estas se encuentran: Asopezca, Asopespa, Cotoca Viva, Asomupa, Fundación Bañó, entre otras; por lo que el 34\% (222) de las viviendas cuentan con al menos un individuo que pertenece a alguna organización local; pero, a pesar de existir organizaciones comunitarias, no hay un trabajo conjunto y articulado entre estas, cada una tiene sus propios objetivos y prioridades, no luchan en conjunto por sacar adelante su territorio, sino que buscan el bienestar de unos pocos, sumiéndose aún más en el atraso y la pobreza mental y física. Para Chacón (2010), el desarrollo comunitario debe ser un proceso educativo destinado a lograr cambios cualitativos en las actitudes y comportamientos de la población, sobre todo en aquellas comunidades en situación de subdesarrollo socioeconómico o de insuficiente utilización de recursos disponibles, a fin de mejorar su calidad de vida. Los individuos pertenecientes a las comunidades deben participar voluntaria, consciente y responsablemente de la solución de los problemas que los aquejen (figura 3 ). 
doi: http://dx.doi.org/10.15359/ree.19-3.6

URL: http://www.una.ac.cr/educare

CORREO: educare@una.cr

\section{Organización comunitaria}

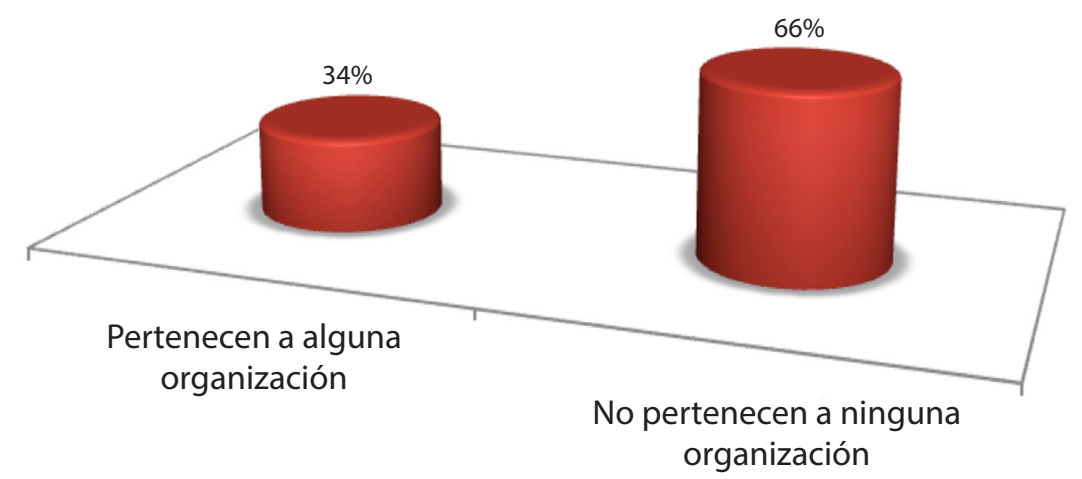

Figura 3. Organización comunitaria. Elaboración propia.

Para el caso de las comunidades asentadas alrededor de los humedales, existe una serie de rivalidades sobre la administración de estos dos cuerpos de agua. Los corregimientos de Palo de Agua y Castilleral señalan que la comunidad de Cotocá Arriba es la que tiene el control y usufructo de estos ecosistemas; que no da cabida a que otras poblaciones contribuyan en la administración de los recursos que proveen los espejos de agua. Por otra parte, si se les pregunta a los habitantes de Cotacá Arriba, ellos afirman que son las otras comunidades las cuales no dan un buen uso a las ciénagas; que la caza y deterioro de esta se debe a las presiones que ejercen las comunidades vecinas y que ellos solo propenden por proteger los recursos del territorio. Básicamente se han pasado los últimos 15 años en esta disputa, mientras tanto y con el trascurrir del tiempo, cada vez se hacen más pobres económica y mentalmente, se deteriora su estructura organizativa y disminuyen las posibilidades de mejorar sus condiciones ambientales. Peor aún, no son capaces de lograr acuerdos, no cuentan con autodeterminación para alcanzar las metas propuestas (si es que las trazan), no trabajan de forma articulada, no definen ni dan prioridad a sus necesidades. Cada vez se hacen más vulnerables en su estructura organizativa, y poco confiables para aquellas organizaciones que quisieran brindarles apoyo, pero por el hecho de estar tan desagregados prefieren no intervenir y sí trabajar con comunidades muy bien organizadas.

Las comunidades aledañas a las ciénagas deben reflexionar sobre cómo se encuentran organizadas y su forma de interactuar. Es necesario desarrollar un proceso eficiente de intervención comunitaria. Al respecto, el Instituto Interamericano para el Desarrollo (2000, citado por Mori, 2008, p. 81) señala que la intervención comunitaria es el conjunto de acciones destinadas a promover el desarrollo de una comunidad a través de la participación activa 
de esta en la transformación de su propia realidad. Por tanto, pretende la capacitación y el fortalecimiento de la comunidad, favoreciendo su autogestión para su propia transformación y la de su ambiente. Dando a la comunidad capacidad de decisión y de acción, se favorece su fortalecimiento como espacio preventivo.

La administración adecuada del territorio va a depender, en gran medida, de la forma como se encuentren estructuradas las comunidades y del grado de desarrollo que presenten tales estructuras. Hernández (2006-2007, p. 286) afirma que:

El desarrollo comunitario es uno de los ámbitos de actuación más valorados dentro de la acción social, ya que permite una intervención en el propio medio de carácter integral y contando con las personas a las que va dirigida tal intervención. La comunidad, compuesta por territorio, población y recursos en constante interacción, se va construyendo con los intereses y aspiraciones de las personas que la componen. La acción conjunta por un objetivo común requiere, eso sí, una metodología que oriente la planificación social y un tejido social dinámico que facilite la organización de la comunidad.

\section{Poca consciencia y compromiso ambiental en las comunidades}

Se estima que el $53 \%$ de las viviendas habitadas cuenta con al menos un individuo que conoce o asocia el término de cultura ambiental al cuidado de la naturaleza; sin embargo, hay un porcentaje alto (47\%) que no tiene conocimiento del término. Del 53\% que conoce el término, el $100 \%$ de este considera que hay escasez de cultura ambiental, de los cuales el $70 \%$ (243) cree que en el territorio son las personas jóvenes y adultas las que tienen mayor carencia de cultura ambiental. Algo irónico es que del total de las 645 viviendas habitadas, todas contaban con al menos un individuo que cree que los recursos naturales existentes en las ciénagas son importantes, otorgándoles un grado de muy importante y más aún, afirma que los problemas ambientales le preocupan mucho.

Igualmente, al preguntar qué tan responsables se sentían por el deterioro ambiental de los humedales, 320 viviendas cuentan con al menos un individuo que no se siente nada responsable por el deterioro de los espejos de agua, que equivalen aproximadamente al $50 \%$ del porcentaje total. Un 33\% se siente poco responsable por la problemática ambiental existente, se estaría hablando que un $83 \%$ de la población no posee un estado de conciencia ambiental que le permita comprender que es parte de una problemática ambiental y cumplir un papel tanto de generadores como de agentes de cambio para la solución de tales problemas existentes. Por otra parte, de las 645 viviendas, un $21 \%$ relaciona el término ambiente a la protección de la naturaleza, otro $29 \%$ a la contaminación y un $24 \%$ a la calidad de vida. Se estima que el $80 \%$ (512) de las viviendas cuenta con al menos un individuo que considera que en sus actuaciones sí tiene consideraciones importantes para el ambiente; un 51\% (327) considera que la actividad 
doi: http://dx.doi.org/10.15359/ree.19-3.6

URL: http://www.una.ac.cr/educare

CORREO: educare@una.cr

humana puede llevar a dañar el ambiente de forma irreversible y un $46 \%$ (298) cree que el deterioro ambiental puede pararse cambiando el modo de vida. Sumando ambos porcentajes, se tiene que el $97 \%$ de encuestados considera que el ser humano puede contribuir a dañar o mejorar las condiciones ambientales de un territorio. Al preguntarle a las personas sobre sus actuaciones hacia el medio, 68\% (441) afirma que generarían acciones positivas hacia el entorno sin importar que otros hicieran algo al respecto, un alto porcentaje de la población estaría dispuesto a tener actitudes y comportamientos proambientales (tabla 2).

Tabla 2

\section{Percepción ambiental}

\begin{tabular}{|c|c|c|c|c|c|}
\hline \multicolumn{6}{|c|}{ PERCEPCIÓN AMBIENTAL } \\
\hline Conoce el término cultura ambiental & \multicolumn{4}{|c|}{ Sí (53\%) } & N0 (47\%) \\
\hline $\begin{array}{l}\text { Cree que hay escasez o carencia de } \\
\text { cultura ambiental en los jóvenes y } \\
\text { adultos de su corregimiento }\end{array}$ & \multicolumn{4}{|c|}{ Sí (100\%) } & № aplica \\
\hline $\begin{array}{l}\text { Basándose en las acciones y } \\
\text { actitudes que toman los habitantes } \\
\text { de su comunidad frente a los } \\
\text { humedales a quiénes les ve más } \\
\text { vacíos en dichos conocimientos }\end{array}$ & Adultos (2.3\%) & \multicolumn{2}{|c|}{ Jóvenes (26.7\%) } & Ambos (70\%) & No aplica \\
\hline $\begin{array}{l}\text { Considera usted que los recursos } \\
\text { naturales presentes en su territorio } \\
\text { tienen alguna importancia }\end{array}$ & \multicolumn{4}{|c|}{ Sí (100\%) } & NO (0\%) \\
\hline $\begin{array}{l}\text { En caso de tener alguna } \\
\text { importancia, qué grado le otorgaría }\end{array}$ & \multicolumn{2}{|c|}{ Muy importante (100\%) } & \multicolumn{2}{|c|}{ Poco importante (0\%) } & Nada importante (0\%) \\
\hline $\begin{array}{l}\text { ¿En qué grado cree que es usted } \\
\text { responsable del deterioro ambiental } \\
\text { de las ciénagas de Baño y Los } \\
\text { Negros? }\end{array}$ & $\begin{array}{l}\text { Muy responsable } \\
\qquad(13,1 \%)\end{array}$ & $\begin{array}{l}\text { Bastante responsable } \\
\qquad(3,8 \%)\end{array}$ & $\begin{array}{l}\text { Poco responsable } \\
(33,5 \%)\end{array}$ & $\begin{array}{l}\text { Nada responsable } \\
(49,6 \%)\end{array}$ & $\begin{array}{l}\mathrm{NS} / \mathrm{NC} \\
(0 \%)\end{array}$ \\
\hline \multirow{2}{*}{$\begin{array}{l}\text { Cuando se habla de ambiente ¿cuál } \\
\text { de los siguientes aspectos es el } \\
\text { primero que se le viene a la cabeza? }\end{array}$} & Contaminación $(29,3 \%)$ & \multirow{2}{*}{$\begin{array}{l}\text { Paisajes agradables } \\
\text { (9\%) }\end{array}$} & \multirow{2}{*}{$\begin{array}{l}\text { Protección de la naturaleza } \\
(21 \%)\end{array}$} & \multirow{2}{*}{$\begin{array}{l}\text { Calidad de vida } \\
\qquad(24,5 \%)\end{array}$} & \multirow{2}{*}{$\begin{array}{l}\text { Desastres naturales } \\
\qquad(7,4 \%)\end{array}$} \\
\hline & Consumo de recursos $(8,8 \%)$ & & & & \\
\hline $\begin{array}{l}\text { ¿Con cuál de estas dos opiniones } \\
\text { está usted más de acuerdo? }\end{array}$ & \multicolumn{3}{|c|}{$\begin{array}{l}\text { Mis actuaciones individuales no tienen consecuencias importantes } \\
\text { para el ambiente }(20 \%)\end{array}$} & \multicolumn{2}{|c|}{$\begin{array}{l}\text { Mis actuaciones individuales tienen consecuencias } \\
\text { importantes para el ambiente }(80 \%)\end{array}$} \\
\hline $\begin{array}{l}\text { ¿Con cuál de las siguientes } \\
\text { opiniones está usted más de } \\
\text { acuerdo? }\end{array}$ & \multicolumn{2}{|c|}{$\begin{array}{l}\text { Normalmente la actividad humana está en armonía } \\
\text { con el ambiente }(3,1 \%)\end{array}$} & \multicolumn{2}{|c|}{$\begin{array}{l}\text { El deterioro ambiental puede pararse cambiando } \\
\text { nuestro modo de vida }(46,2 \%)\end{array}$} & $\begin{array}{l}\text { La actividad humana puede } \\
\text { llevarnos a dañar el ambiente } \\
\text { de forma irreversible }(50,7 \%)\end{array}$ \\
\hline $\begin{array}{l}\text { ¿Cuál de las siguientes actuaciones } \\
\text { refleja mejor su situación personal } \\
\text { en relación con el ambiente? }\end{array}$ & $\begin{array}{l}\text { Intento actuar generando } \\
\text { acciones positivas hacia el } \\
\text { medio, pero solo funciona } \\
\text { si otros actúan también } \\
(21,4 \%)\end{array}$ & $\begin{array}{l}\text { Intento actuar sin } \\
\text { importarme lo que hagan } \\
\text { otros } \\
(68,4 \%)\end{array}$ & $\begin{array}{l}\text { No lo intento porque otros } \\
\text { tampoco lo hacen } \\
(2,6 \%)\end{array}$ & $\begin{array}{l}\text { No sé qué hacer } \\
(7,6 \%)\end{array}$ & $\begin{array}{l}\text { No me preocupa el ambiente } \\
(0 \%)\end{array}$ \\
\hline
\end{tabular}

Nota. Elaboración propia. 
Lo interesante de la tabla 2, y que resalta a simple vista, es cómo las poblaciones encuestadas le dan un alto grado de importancia a los recursos presentes en su territorio y cómo les preocupan mucho los problemas ambientales, aun cuando un porcentaje tan alto (83\%) de los habitantes no se sienten responsables del deterioro de las ciénagas, sin omitir que el $68 \%$ de las personas se identifican con la generación de acciones positivas al medio sin necesidad de que las demás actúen; además, un $46 \%$ cree que cambiando el modo de vida se puede para el deterioro ambiental (46\%) y otro $51 \%$ considera que la actividad humana puede dañar de forma irreversible el ambiente. De ser coherentes y conscientes de lo que dicen: ¿Por qué el deterioro de los humedales va en aumento? ¿Realmente a las personas les interesa proteger los humedales? ¿Cuál es el costo que están dispuestos a pagar por proteger estos ecosistemas? ¿Cuán importantes son las variables económica, educativa y organizativa de las comunidades en toda esta ecuación?

\section{Inoperancia de las autoridades ambientales}

La percepción que tienen las poblaciones vecinas de los humedales acerca de las autoridades ambientales y locales y de su gestión para la solución de los problemas ambientales del territorio es bastante negativa. De las 645 viviendas se estima que el $66 \%$ cuenta con al menos un individuo que considera que la situación ambiental en el territorio ha empeorado con el pasar del tiempo y un $24 \%$ (158) afirma que a la fecha sigue igual la problemática ambiental en los humedales. Un $71 \%$ de las viviendas cuenta con al menos una persona que cree que Colombia, en comparación con otros países que protegen el ambiente, hace menos de lo suficiente para cuidarlo.

Ante la débil presencia de las autoridades ambientales con programas para la protección de los humedales y el poco control y seguimiento que se le hace a los ecosistemas en mención, es muy fácil que se deterioren, pues existen pobladores que saquean estos espejos de agua y sus alrededores $y$, ante la mirada cómplice de algunos habitantes, terminan cazando y negociando la fauna existente, entre otras tantas acciones. No se omite que, particularmente, los pocos habitantes que se atreven a intervenir para evitar este tipo de acciones terminan siendo amenazados y, para cuando las autoridades ambientales y policías Ilegan, ya es demasiado tarde e impera la ley del silencio.

Básicamente, al preguntar a las personas encuestadas acerca de qué factores estaban influenciando en el deterioro de los humedales, se obtuvo que un 55\% (354) de las viviendas cuenta con al menos un individuo que cree que la destrucción de los humedales obedece a una sinergia de factores relacionados con la poca cultura ambiental de las poblaciones, la falta de gestión de las autoridades ambientales nacionales y locales, y la pobreza y pocas oportunidades de empleo en la región. Si bien una gestión ambiental eficiente es el resultado de un trabajo conjunto 
doi: http://dx.doi.org/10.15359/ree.19-3.6

URL: http://www.una.ac.cr/educare

CORREO: educare@una.cr

entre autoridades ambientales y las comunidades, los habitantes de la zona no perciben que la corporación ambiental de los valles del Sinu y San Jorge (CVS) los apoyen o esté verdaderamente interesada en desarrollar acciones conjuntas para la protección de los ecosistemas; señalan que las pocas veces que la organización hace presencia en el área de estudio responde más a un requisito para cumplir metas institucionales que a la búsqueda de verdaderas soluciones a los problemas ambientales existentes y que, sobre todo, afecta a las comunidades vecinas, de allí que las poblaciones noten la poca funcionalidad de la corporación ambiental en el territorio (figura 4).

De las 645 viviendas habitadas, se estimó que 187 contaban al menos con un individuo que por lo menos consideró que dentro de las medidas más eficaces en orden de importancia para la solución de la problemática antes descrita se encontraba el desarrollo de programas de educación ambiental con un 29\%, 155 referían con al menos un individuo que consideró la implementación de leyes más estrictas representado en un $24 \%$ y 141 tenían a por lo menos un individuo que indicó como mecanismo para la solución de la problemática ambiental la aplicación de multas a los causantes de los daños, consolidando un $22 \%$. Todas estas medidas, creen las poblaciones vecinas de los humedales, deberían ser implementadas con prontitud por parte de las Corporación Ambiental y la administración local, de no ser así, la problemática ambiental llegaría a un punto de no retorno.

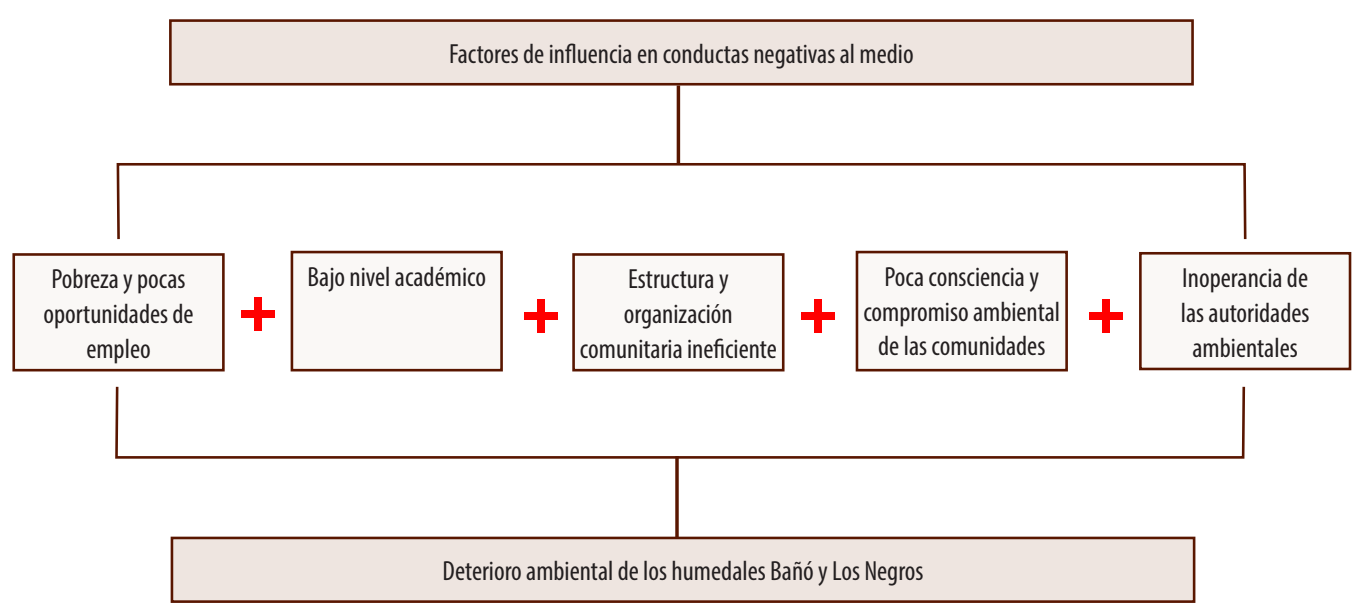

Figura 4. Factores de incidencia en conductas negativas al medio. Elaboración propia.

\section{Conclusiones}

Esencialmente el deterioro de los humedales Bañó y Los Negros está relacionado con la sinergia de una serie de factores dentro de los que se encuentran los bajos ingresos económicos y nivel de educación, poca organización comunitaria, inoperancia de las autoridades ambientales y la falta de conciencia de las comunidades para la protección de los recursos naturales presentes en su territorio. Lo anterior ha contribuido a que tales ecosistemas, con el pasar del tiempo, se degraden hasta sus límites, perdiendo su importancia ecológica. 
Las autoridades ambientales locales y las comunidades deben ejercer un mayor control en el manejo de los recursos ambientales. Es vital para las comunidades asentadas alrededor de los humedales desarrollar procesos de formación ambiental, preferiblemente de tipo no formal, con el propósito de generar actitudes positivas hacia estos ecosistemas por parte de sus habitantes, como también a través de tales procesos educativos formular estrategias productivas y amigables con el ambiente. De la forma como se organicen las poblaciones dependerá, en gran medida, su capacidad de desarrollo y supervivencia, así como la mejora de las condiciones ambientales y de recursos económicos.

\section{Recomendaciones}

A partir de la identificación de los factores que están incidiendo en las conductas ambientales negativas hacia los humedales de Bañó y Los Negros, podría generarse un modelo educativo no formal de educación ambiental que contribuya a mejorar la relación ser humano-medio.

Es necesario que las poblaciones vecinas de los humedales desarrollen un proceso de intervención comunitaria, a fin de estructurarse y organizarse de forma eficiente, y así poder implementar soluciones a las problemáticas ambientales que han generado y contribuido al deterioro de sus condiciones de vida; además, que se implementen acciones encaminadas a mejorar el nivel de educación de las comunidades vecinas de los espejos de agua, a fin de que se reduzca el porcentaje de personas con niveles incompletos de estudios básicos, mejorando su capacidad de apropiación de conocimientos, valores y la capacidad de pensamiento crítico-reflexivo.

\section{Referencias}

Álvarez, P. y Vega, P. (2009). Actitudes ambientales y conductas sostenibles. Implicaciones para la educación ambiental. Revista de Psicodidactica, 14(2), 245-260. Recuperado de http://www. redalyc.org/pdf/175/17512724006.pdf

Chacón, M. D. (2010). El desarrollo comunitario. Revista Innovación y Experiencias Educativas, 29, 1-11. Recuperado de http://www.csi-csif.es/andalucia/modules/mod ense/revista/pdf/ Numero 29/M DOLORES CHACON 1.pdf

Colín, L. (Julio-Septiembre, 2003). Deterioro ambiental vs. desarrollo económico y social. Boletin IIE. Recuperado de http://www.iie.org.mx/boletin032003/art2.pdf

DANE. (18 de abril de 2013). Pobreza monetaria y multimensional en Colombia 2012. Boletín de Prensa. Recuperado de http://www.dane.gov.co/files/investigaciones/condiciones vida/pobreza/ boletin pobreza 2012xx.pdf

Galarza, E. y Gómez, R. (s. f.). Análisis económico de los problemas ambientales urbanos. Recuperado de http://info.worldbank.org/etools/docs/library/112219/peru/docs/Modulolll/Galarza\%20 Gomez\%20An\%E1lisis\%20Medio\%20Ambiente.pdf 
doi: http://dx.doi.org/10.15359/ree.19-3.6

URL: http://www.una.ac.cr/educare

CORREO: educare@una.cr

García, L., Figueredo, L. y Gómez, C. (Mayo, 2013). Desarrollo e implementación de un programa de educación ambiental "proyecto comunitario" para concientizar a la población de Lagos de Moreno. Revista Pistas Educativas, 101, 198-206. Recuperado de http://pistaseducativas.itc.mx/ wp-content/uploads/2013/06/18-GARCIA-PE-101-198-206.pdf

Hernández, P. J. (2006-2007). Educación y desarrollo comunitario: Dialogando con Marco Marchioni. Cuestiones pedagógicas, 18, 285-300. Recuperado de http://institucional.us.es/revistas/ cuestiones/18/19\%20educacion\%20y\%20desarrollo.pdf

Holahan, C. J. (1991). Psicología ambiental: Un enfoque general. México: Limusa.

Hwang, Y.-H., Kim, S.-I y Jeng, J.-M. (2000). Examining the Causal Relationship Among Selected Antecedents of Responsable Environmental Behavior [El examen de la relación causal entre antecedentes seleccionados de comportamiento ambiental responsable]. The Journal of Environmental Education, 31(4), 19-25. doi: http://dx.doi.org/10.1080/00958960009598647

Instituto Geográfico Austin Codazzi. (1994). Fotografía aérea de las Ciènagas de Bañó y Los Negros. Bogotá: Autor.

Martínez, R. (Enero-Junio, 2010). La importancia de la educación ambiental ante la problemática actual. Revista Electrónica Educare, 14(1), 97-111. Recuperado de http://www.redalyc.org/ pdf/1941/194114419010.pdf

Meadows, D., Randers, J., Meadows, D. y Behrens, W. (1972). Los límites del crecimiento. New York: Editorial Signet Books.

Mori, M. (2008). Una propuesta metodológica para la intervención comunitaria. Liberabit, 14, 81-90. Recuperado de http://www.scielo.org.pe/pdf/liber/v14n14/a10v14n14.pdf

\section{Cómo citar este artículo en APA:}

Villadiego-Lorduy, J., Huffman-Schwocho, D. P., Guerrero-Gómez, S. Y., Cortecero-Bossio, A. y Rivero-Espinoza, S. (Setiembre-Diciembre, 2015). Factores de incidencia de conductas ambientales negativas hacia las ciénagas de Bañó y Los Negros. Revista Electrónica Educare, 19(3), 1-16. doi: http://dx.doi.org/10.15359/ree.19-3.6

Nota: Para citar este artículo en otros sistemas puede consultar el hipervínculo "Como citar el artículo" en la barra derecha de nuestro sitio web: http://www.revistas.una.ac.cr/index.php/EDUCARE/index 